\title{
Hyperthyroidism during pregnancy due to coexistence of struma ovarii and Graves' disease
}

\author{
Annie W.C. Kung, John T.C. Ma, Christina Wang and Rosie T.T. Young \\ Department of Medicine, University of Hong Kong, Queen Mary Hospital, Hong Kong.
}

\begin{abstract}
Summary: A patient with hyperthyroid Graves' disease and struma ovarii is described. She had pre-existing Graves' disease and positive thyrotrophin receptor antibody. She was treated with radioactive iodine 5 months before she became pregnant. Paripartum she had torsion of an ovarian cyst with histological evidence of a functional struma ovarii. Immediate exacerbation of her thyrotoxic state was observed after operation as a result of release of thyroid hormone from the tumour. It is postulated that the tumour was stimulated by circulating thyrotrophin receptor antibody.
\end{abstract}

\section{Introduction}

Struma ovarii causing hyperthyroidism is a rare clinical condition. The diagnosis is usually suspected in a clinically hyperthyroid patient with negative scintiscan of the neck, who has no history suggestive of thyroiditis, iodine contamination or iatrogenic thyroid hormone consumption. We report here a unique patient with relapse of hyperthyroidism during pregnancy due to coexistence of struma ovarii and Graves' disease.

\section{Case report}

A 40 year old woman was referred to our hospital for management of recurrence of her hyperthyroidism. She had a history of diffuse thyroid enlargement and hyperthyroidism 4 years before and was treated with anti-thyroid drugs for one year with clinical remission. On this presentation she had recurrence of palpitation, marked sweating and weight loss for 2 months together with increase in goitre size. There was no family history of thyroid or other autoimmune disease. Examination revealed tachycardia, diffuse moderately enlarged thyroid gland and no eye signs. Total T4 (TT4) was $258 \mathrm{nmol} / 1$ (normal 62 to 154), with free thyroxine index (FTI) of 297 (normal 56 to 152) and free T4 (FT4) of $54.7 \mathrm{pmol} / 1$ (normal range 9.9-25.9). TSH was undetected by sensitive immunoradiometric assay (Boots-Celtech). There was diffuse increased uptake at the neck on ${ }^{131} I$ scan with a 4-hour thyroidal uptake of $70 \%$ (normal 12 to 45 ). She was treated with $326 \mathrm{MBq}$ of radioactive

Correspondence: A.W.C. Kung, M.R.C.P. (UK). Accepted: 8 June 1989 iodine and also advised to practise contraception.

She became euthyroid 3 months afterwards but returned 2 months later with a relapse of symptoms and was also found to be pregnant. TT4 then was $307 \mathrm{nmol} / 1$ with a FTI of 203. The thyrotrophin receptor antibody ${ }^{1}$ was positive at $23.8 \%$ (normal $<10 \%$ ). Carbimazole was given and the patient subsequently remained euthyroid and the drug was withdrawn at 38 weeks of gestation. Ultrasonagraphy (at 18 weeks of gestation) showed a normal fetus with no abnormal pelvic mass.

At 41 weeks of gestation, the patient developed sudden onset of severe right lower quadrant abdominal pain. This was associated with signs of fetal distress. Laparotomy showed torsion of a haemorrhagic right ovarian cyst of $5 \times 4 \mathrm{~cm}$ diameter. There was no ascites and ovarian cystectomy was performed. The fetus was delivered by Caesarean section and was normal without any thyroid enlargement. Thyroid function test of the patient one day after delivery showed marked elevation of TT4 to $1580 \mathrm{nmol} / \mathrm{l}$ and FTI of 1254 . Carbimazole and propranolol were reinstituted with rapid improvement of symptoms and 2 weeks later, the patient was rendered biochemically euthyroid.

Histology of the ovarian cyst showed multiple colloid-containing follicles with positive immunoperoxidase staining for thyroglobulin and thyroxine. Some follicles were markedly distended by colloid with attenuation of epithelium. There were no other teratomatous or germ cell elements. The finding was that of struma ovarii.

In view of the diagnosis of struma ovarii, carbimazole was stopped to assess the possibility of remission after tumour removal. Total body ${ }^{131}$ I scintiscan performed 4 weeks after stopping car- 
bimazole showed normal thyroidal uptake and there was no abnormal area of increased uptake over the rest of the body. However, the patient became biochemically hyperthyroid again 6 months after delivery together with diffusely elevated thyroidal uptake on ${ }^{131}$ I scintiscan. Thyrotrophin receptor antibody was also positive at $25.6 \%$. In view of relapse of her Graves' disease, a second dose of radioactive iodine was given and she remained euthyroid for a follow-up period of 2 years.

Thyroid function of the baby has remained normal since birth. Thyrotrophin receptor antibody was elevated in the cord blood at $29.0 \%$, which subsequently decreased to $18.1 \%$ at one week and became normal at one month of age.

\section{Discussion}

Struma ovarii is a teratoma of the ovary in which thyroid tissue is present exclusively or forms a grossly recognizable component of a more complex teratoma. The incidence of hyperthyroidism due to functional thyroid tissue within the tumour is 5 to $15 \%$ of the total reported cases in the literature. ${ }^{2,3}$ Many of the reported cases do not have precise thyroid function tests. The problem is also complicated by a high incidence of goitres of $41.7 \%$ in patients with struma ovarii and hyperthyroidism. ${ }^{4}$

The diagnosis of a hyperfunctional struma ovarii prior to surgery had been described by high ${ }^{131} I$ uptake in the pelvis associated with low radioiodine uptake in the neck, together with clinical and biochemical evidence of hyperthyroidism. ${ }^{4,5}$ Hasleton et al. ${ }^{6}$ have also demonstrated the functional status of the tumour by positive immunoperoxidase staining for thyroglobulin and triiodothyronine. The evidence for a functional struma in our case was a positive immunoperoxidase staining for thyroxine and a striking hyper-

\section{References}

1. Rees Smith, B. \& Hall, R. Thyroid stimulating immunoglobulins in Graves' disease. Lancet 1974, ii: 427-431.

2. Marcus, C.C. \& Marcus, S.L. Struma ovarii. A report of seven cases and a review of the subject. Am J Obstet Gynecol 1961,81: $752-761$.

3. Bortolozzi, G. Lo struma ovarico presentazione di 5 casi e rassegna della letteratura. Annali din Obstetricia e Ginecologia 1967, 89: 310-331.

4. Brown, W.W., Shetty, K.R. \& Rosenfeld, P.S. Hyperthyroidism due to struma ovarii: demonstration by radioiodine scan. Acta Endocrinol (Copenh) 1973, 73: 266-272.

5. Yeh, E.L., Meade, R.C. \& Ruetz, P.P. Radionuclide study of struma ovarii. J Nucl Med 1973, 14: 118-121. thyroxinaemia associated with ovarian torsion. Thyroidal scan was not performed in view of the pregnancy.

The role of the thyroid gland in the production of the hyperthyroid state has not been evaluated satisfactorily in those patients whose symptoms have been ascribed solely to their ovarian struma. In our patient, definitive diagnosis of Graves' disease is supported by the diffuse increased uptake by the thyroid gland on ${ }^{131}$ I scintiscan and elevated thyrotrophin receptor antibody, thus additional source of ectopic hormone production was not suspected and the diagnosis of struma ovarii was not made before operation. The hyperthyroid state of the patient at all times during pregnancy probably resulted from the Graves' disease and eutopic thyroid overfunction. We believe that the production of thyrotrophin receptor antibody stimulated both the thyroid gland and the struma ovarii to produce thyroid hormones, as has been suggested by Lefort et al. ${ }^{7}$ Torsion of the tumour led to release of preformed hormone and leading to markedly elevated serum thyroxine level. It was possible that only a very small tumour was present at early stage of pregnancy which was not detectable by ultrasonogram. The growth of the tumour could possibly be stimulated by pregnancy and thyroid growth stimulating antibody, which has been reported to be present in $85 \%$ of patients with active Graves' disease. ${ }^{8}$ Unfortunately it has not been possible to examine the effect of thyrotrophin receptor antibody in the patient's serum on the tumour growth and differentiation in vitro to verify our hypothesis.

\section{Acknowledgement}

The authors thank Dr V. Chan for the supply of thyroxine antibody and Ms P. Ho for technical assistance.

6. Hasleton, P.S., Kelehan, P., Whittaker, J.S., Burslem, R.W. \& Turner, L. Benign and malignant struma ovarii. Arch Pathol Lab Med 1978, 102: 180-184.

7. Lefort, G., Commenges-Ducos, M., Denechaud, M., Rivel, J. \& Latapie, J. Goitre ovarien au cours d'une maladie Basedow. La Nouvelle Presse Medicale 1981, 10: 2209-2210.

8. Valente, W.A., Vitti, P., Rotella, C.M. et al. Antibodies that promote thyroid growth - a distinct population of thyroidstimulating autoantibodies. $N$ Engl J Med 1983, 309: $1028-1034$. 